\title{
Diphtheria in Iran
}

\author{
BY IRAJ ZAMIRI AND M. G. McENTEGART \\ Department of Medical Microbiology, University of Sheffield, \\ Sheffield S102TN \\ AND ALICE SARAGEA \\ Institute Dr I. Cantacuzino, Bucharest, Romania
}

(Received 17 March 1972)

\begin{abstract}
SUMMARY
A study of diphtheria in Iran was undertaken during the summer of 1969 . Diphtheria was found to be more common among the younger people and to reach its peak 3 months after the start of the school term, during the coldest period of the year. Owing to the liberal use of antibiotics a number of modified clinical cases were observed. Of the strains isolated, $89.7 \%$ were gravis, $1.3 \%$ intermedius and $9 \%$ mitis. Phage typing of these strains showed that the mitis and intermedius strains could only be typed by adapted phages and the majority of gravis strains were phage type XIV, which is the epidemic type seen in other countries.

As the mass immunization campaign reduces the incidence of diphtheria in Iran, the epidemiological pattern of the disease will change as seen in the U.K. and U.S.A. It is, therefore, suggested that in the future further studies of diphtheria in Iran be undertaken in order to provide us with information about the changing epidemiological pattern of the disease.
\end{abstract}

\section{INTRODUCTION}

Although the epidemiological pattern of diphtheria in the world is rapidly changing, the disease still remains a significant health problem in many countries (Editorial, 1970). Better standards of living, mass immunization, improved diagnosis, prompt treatment, and more effective carrier tracing have within the last decade reduced the incidence of diphtheria in the remaining European foci. South American, African and Asiatic countries are now taking similar steps.

While the number of doctors in Great Britain who have had experience of diphtheria in either its clinical or laboratory aspects continues to dwindle, the risk of the disease being imported into the U.K. and occurring in classical or modified form remains (Zamiri, 1970). With these problems in mind, an investigation of diphtheria in Iran was undertaken and some first-hand experience of the disease obtained. Over 200 Iranian strains of $C$. diphtheriae were brought back so that their biotypes and phage types could be studied.

The programme included a study of clinical diphtheria in Iran during July, August and September 1969, followed by the examination of the strains of C. diphtheriae in Sheffield. During the latter stage of the investigation Dr A. 


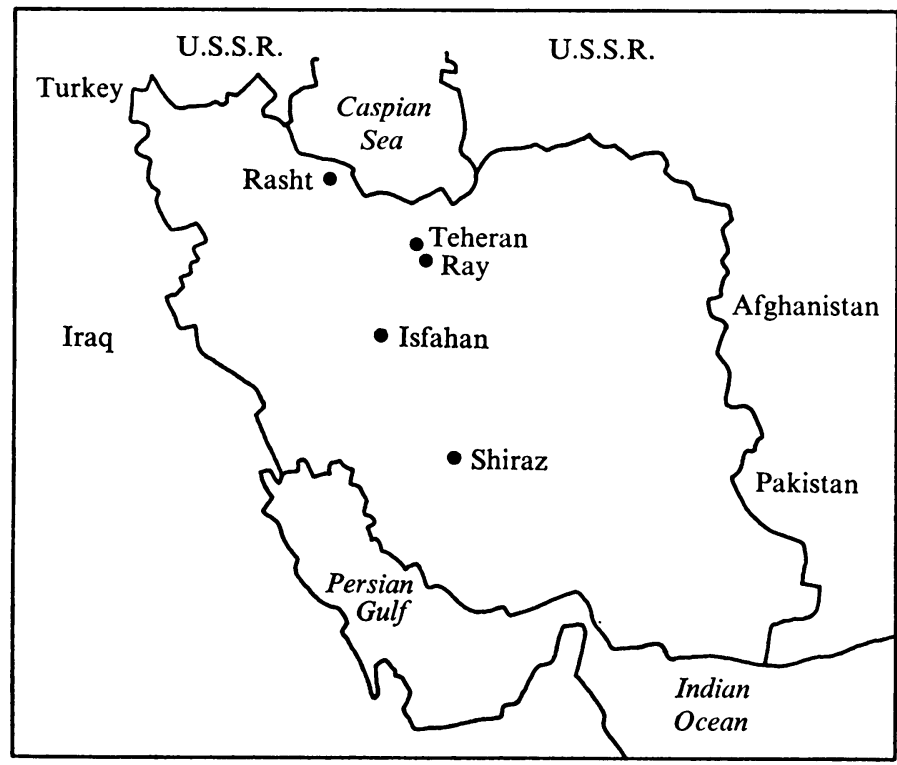

Fig. 1. Map of Iran, showing areas visited during this study.

Saragea came to Sheffield in order to demonstrate and organize the phage-typing methods which had been developed in her laboratory in Romania.

\section{Area studied}

The map of Iran and neighbouring countries (Fig. 1) shows the cities (Teheran, Ray and Rasht) where the clinical cases were seen and those (Teheran, Ray, Isfahan and Rasht) from which strains of C.diphtheriae had been isolated in earlier surveys.

\section{The incidence of diphtheria in Iran}

Examination of the W.H.O. statistical reports (1960-4) on diphtheria in Iran showed, in contrast to most other countries, a rising incidence of the disease (Fig. 2). Further investigation of this rise, which had occurred despite an increased immunization rate, showed some contradictions (as, for example, a relatively low death rate $-2 \cdot 2 \%$ ) compared with another report from Iran (Tahernia \& Motamed, 1969) suggesting that the figures given to W.H.O. exaggerate the incidence of the disease, or immunization was too late to prevent this epidemic. Another influencing factor is the rapidly growing population, a large proportion of whom were not protected (Fig. 2). Considering the rapid rate of population increase, the falling incidence of diphtheria in Iran is more dramatic than it may appear (Fig. 2).

General medical progress in Iran led to a greater awareness of the problem of diphtheria. As the rate of development of laboratory facilities to confirm the diagnosis had not kept pace, the use of clinical criteria alone was likely to lead to an exaggeration of the incidence. The mass immunization campaign which started in 1965 is showing good results (Fig. 2). In Teheran, with a population of nearly 3 million, the annual incidence of diphtheria, formerly about 2500 cases, has been 


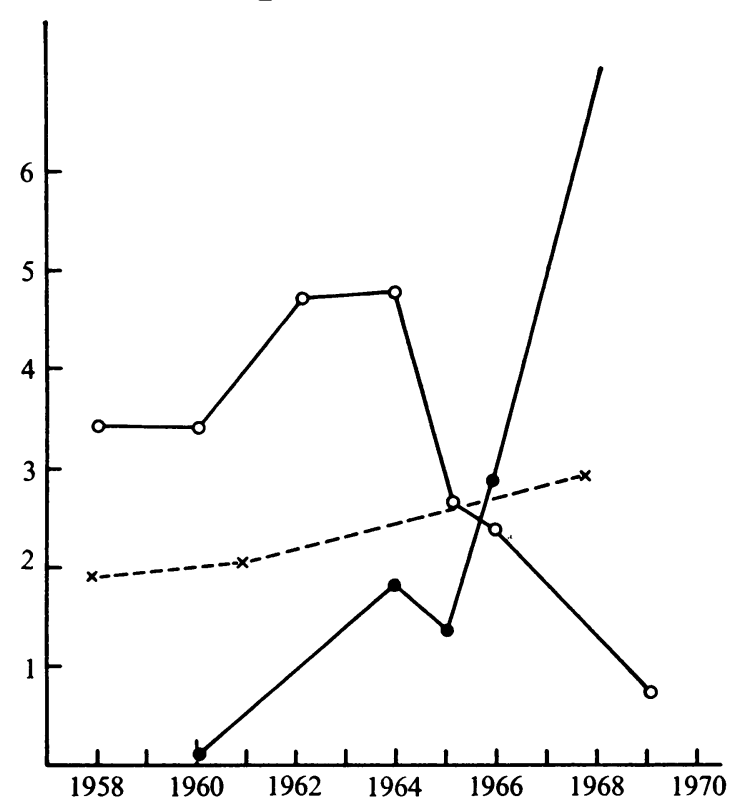

Fig. 2. Annual incidence of diphtheria, immunization rate, and population increase in Iran (1958-69). $\bigcirc-\bigcirc$, Incidence of diphtheria, in thousands; -0 , immunization rate, in millions; $x---x$, population, in 10 millions.

reduced to a fraction of that number (H. Mirchamsy, pers. comm.) From 1965 to 1968, over 7 million people, especially in rural areas, were immunized (Iranian Health Corps Report, 1969). The effect of immunization is clearly seen in this report, which shows that in 1969 the incidence in the peak months of January/ February was one-seventh of that for the same months before the mass immunization campaign (1964).

\section{The seasonal incidence of diphtheria}

The variation in the seasonal incidence in Iran, with the peak in the winter (Fig. 3) is very similar to that seen in diphtheria epidemics in other countries. This seasonal variation becomes less marked as the epidemic incidence of the disease gives place to the endemic incidence. The start of the school year in September may also contribute to the rising incidence of carriers and cases in the winter season (Fig. 3).

\section{Carrier rate}

There is only one report on the incidence of diphtheria bacilli in routine throat and nose swabs in Iran. Esterabady, Taslimi \& Nategh (1963) reported that 200 $(33 \%)$ of 600 nose and throat swabs sent to the Razi and Pasteur Institutes in Teheran were positive for $C$. diphtheriae. Unfortunately, they do not comment on the selection of their patients, but it is probable that they were all suspected cases of diphtheria. If this is so, the figure of $33 \%$ does not represent the true incidence of carriers. 


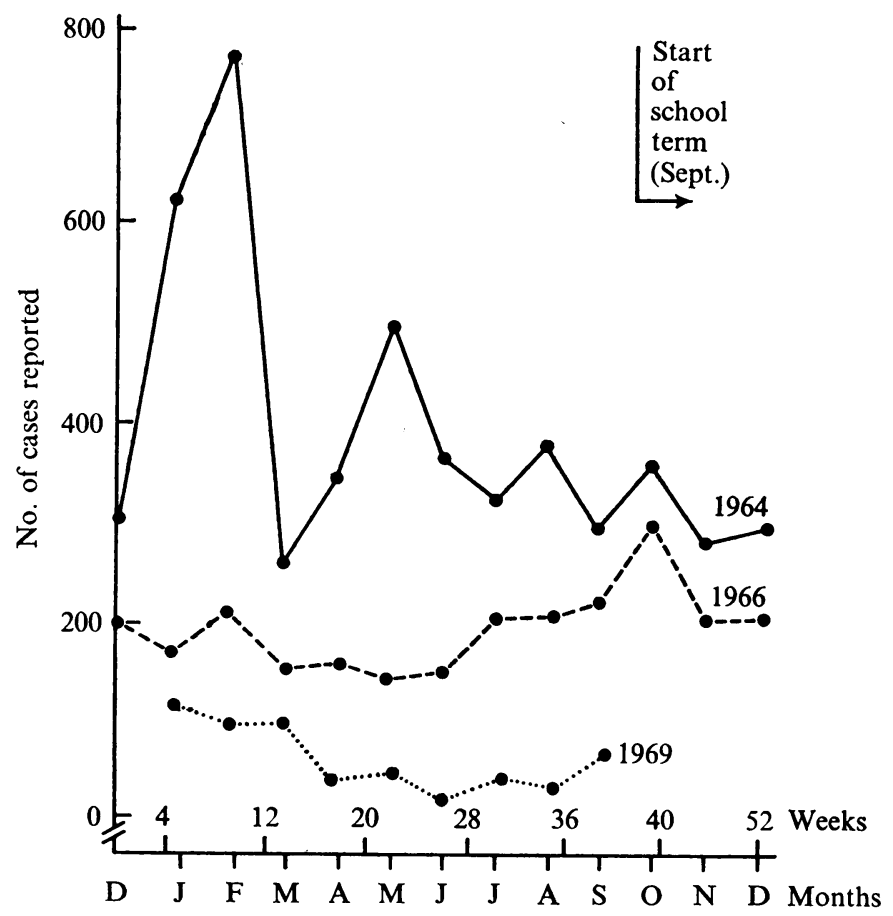

Fig. 3. Monthly incidence of diphtheria in Iran during 1964, 1966 and 1969.

\section{Age incidence}

Throughout the world classical diphtheria is still mainly a disease of children. Tahernia \& Motamed (1969) confirmed this for Shiraz, where $62 \%$ of the 95 cases studied occurred in children below the age of 6 . However, during the field study which was carried out in the north, only five $(20 \%)$ of the 25 cases seen were in this age group. This difference may be explained by the school holiday accentuating the season of minimal incidence and possibly by variation due to areas studied. It does seem likely that school children carry the infection to the pre-school age siblings. In many under-developed countries children live in crowded conditions, and during the colder season, sleep with their elders. Such close contacts among the members of one family, often undernourished and suffering from respiratory infections, must contribute to the rising incidence.

\section{MATERIALS AND METHODS}

Nine of the strains of $C$. diphtheriae studied in this survey were isolated from the 25 clinical cases seen during the field study in Iran. The low isolation rate may be due to the early use of antibiotics. The remaining strains, most of which were lyophilized, were provided through the kindness of colleagues in Teheran and Isfahan. All were isolated from cases of clinical diphtheria. The total number of strains examined was 232 .

All strains were typed according to the criteria of McLeod (Anderson, Happold, McLeod \& Thompson, 1931; Anderson, Cooper, Happold \& McLeod, 1933). The 
Table 1. Biotypes of 232 strains of C. diphtheriae isolated in Iran

\begin{tabular}{|c|c|c|c|c|c|c|}
\hline \multirow[b]{2}{*}{ Total no. } & \multicolumn{2}{|c|}{ Gravis } & \multicolumn{2}{|c|}{ Intermedius } & \multicolumn{2}{|c|}{ Mitis } \\
\hline & No. & $\%$ & No. & $\%$ & No. & $\%$ \\
\hline 232 & 208 & $89 \cdot 7$ & 3 & 1.3 & 21 & 9 \\
\hline
\end{tabular}

toxigenicity of the first 20 , and all subsequent strains giving equivocal results by in vitro tests, were confirmed by their lethal effect on guinea-pigs. Each strain was phage typed by the method of Saragea \& Maximescu (1966). A few minor changes in their method were made in order to make use of commercial media available in the United Kingdom. The strain for phage typing was grown overnight on a Loeffler slope and the whole of the growth from this culture was used to inoculate a $3 \mathrm{ml}$. volume of Lab-Lemco broth containing $\mathrm{CaCl}_{2}$ and cystine. This was broth incubated at $35^{\circ} \mathrm{C}$ for $1 \frac{1}{2} \mathrm{hr}$. and was then used to seed the entire surface of a heart brain agar plate (heart brain agar was prepared by incorporating $1 \%$ Oxoid agar in heart brain broth (Difco)). Seeded plates were dried in the incubator for $40 \mathrm{~min}$. The basic set of 24 diphtheria phages (prepared by Drs Saragea and Maximescu in Bucharest) were used at concentrations of 100 RTD. The phage types were determined according to the lytic 'patterns' described in the original phagetyping scheme in which all degrees of lytic reactions were taken into account.

The phage samples were applied initially by Pasteur pipettes, but later use was made of a modified P.H.L.S. phage applicator (Lidwell, 1959).

Strains which were, at this point, untypable were tested by a series of 'additional' phages which consisted of a further set of 33 adapted phages from the Romanian Diphtheria Laboratory. A few remaining strains, which were still untypable, were further investigated by adapting the lysogenic phage, 951, to one of them and then testing this adapted phage (951/9) against the other untypable strains. Thus it was possible to show that the untypable strains behaved in the same way when tested with the adapted phage 951/9.

\section{Biotyping}

\section{RESULTS}

Of the 232 strains examined, $89 \cdot 7 \%$ were gravis, $9.0 \%$ mitis and $1.3 \%$ intermedius (Table 1). Three of the gravis and one of the mitis varieties were nontoxigenic.

\section{Phage typing}

All but eight of the strains were phage typable. About $95 \%$ of the gravis toxigenic strains fell into eight phage types (Table 2); among these $53 \%$ were phage type XIV, $36 \%$ were phage type XVI. The remainder were distributed amongst phage types XIV ' $a$ ' and ' $b$ ', VI, XVII, XVIII and XIX.

The intermedius and mitis strains were typable, with the adapted phages 951/9 and 951/553. Some Albanian and French strains have been found to be of this phage type.

The biotypes were in general agreement with the phage types apart from two 
Table 2. Phage types of 232 strains of C. diphtheriae isolated in Iran

$\begin{array}{ccc}\text { Biotype } & \text { 'Phage type } & \text { No. of } \\ \text { strains }\end{array}$

strains which behaved atypically in that they were morphologically and biochemically mitis, but were typed by gravis phages (type XIV).

Lysogenicity of some of the strains was also studied. Eleven strains tested proved to be lysogenic and, as expected, all these were toxigenic.

\section{DISCUSSION}

In Iran, improved health services plus the massive immunization programme which began in 1965, have brought about a rapid fall in the incidence of diphtheria. As might have been predicted, the major fall has been during the peak period, but this fall has also been very significant in other seasons.

As the disease becomes less common, it is likely that the pattern of diphtheria may soon be similar to that seen in more developed countries, a much lower annual incidence with a proportionately higher death-rate. The higher death-rates in the western countries and in U.S.A. may be due to the modification by antibiotics of the classical picture of diphtheria, plus a general unfamiliarity with the disease.

The change in the age incidence of diphtheria in children as a result of the immunization campaign, social betterment, etc., was seen as a relative increase of cases in the 5- to 10-year-old group and a decrease in the 0- to 5-year-old group. This change was the same as that seen in the U.K. between 1900 and 1935.

Among the 232 strains studied, two toxigenic gravis strains each produced two distinctive types of colony on tellurite medium, which although biochemically indistinguishable proved to be of different phage types. This may represent a double infection. The culture from one person showed both mitis and gravis biotypes, which were also different in phage typing. The mitis variety was typable by the adapted phage I (951/9), but the gravis variant was morphologically and biochemically a toxigenic gravis and phage typed as group VI. Once more, this raises the question of a mixed infection (or mixed cultures).

The most common phage type was type XIV. This type has been found in most of the European countries, in Australia and U.S.A. and it also played a major part in the 1956-64 epidemic in Romania, when it was the commonest epidemic type. Type XVI, the next most common type, has also been found in Europe, Australia, Canada, U.S.A., etc. Four of the five examples of phage type VI, more 
commonly seen among the carriers, were isolated in Isfahan, whereas only $20 \%$ (48) of the strains examined were from that region. The two strains isolated in Rasht were of the same phage type (XIV) and biotype (gravis).

The biotypes and phage types of the 232 Iranian strains of $C$. diphtheriae which were isolated from centres investigated were those associated with epidemic outbreaks of the disease in Europe.

A preponderance of toxigenic gravis strains is characteristic of epidemic diphtheria. The phage typing and biotyping of strains from Iran followed this expected pattern and was in general agreement with the Australian study described by Gibson, Cooper, Saragea \& Maximescu (1970). As has been shown in most countries studied, the climate, social conditions and state of immunity play an important part in the epidemiology of the disease, especially as the main prevalence is in children. Recent antidiphtheria measures in Iran are showing results. There has already been a rapid fall in the incidence of diphtheria in the larger towns and a similar fall in rural areas should follow. These developments provide an ideal situation for the epidemiological study of diphtheria.

We should like to thank the Wellcome Trust for supporting the study, Drs Mirchamsy, Siyadet, Zafari, Motamed and Hazeghi for their help during the field study, Dr Paula Maximescu for adapting phage 951, and Dr W. H. H. Jebb.

\section{REFERENCES}

Anderson, J. S., Happold, F. C., McLeod, J. W. \& Thompson, J. G. (1931). On the existence of two forms of diphtheria bacillus - B. diphtheriae gravis and B. diphtheriae mitis - and a new medium for their differentiation and for the bacteriological diagnosis of diphtheria. Journal of Pathology and Bacteriology 34, 667-81.

Anderson, J. S., Cooper, K. E., Happold, F. C. \& McLeod, J. W. (1933). Incidence and correlation with clinical severity of gravis, mitis and intermediate types of Diphtheria bacillus in a series of 500 cases at Leeds. Journal of Pathology and Bacteriology 36, 169-82.

Editorial (1970). Diphtheria. Lancet i, 1215.

Esterabady, H., Taslimi, H. \& Nategh, R. (1963). Bacteriological typing of $C$. diphtheriae strains recently isolated in Teheran. Acta Medica Iranica 6, 22-32.

Gibson, L. F., Cooper, G. N., Saragea, A. \& Maximescu, P. (1970). A bacteriological study of strains of $C$. diphtheriae isolated in Victoria and New South Wales. Medical Journal of Australia i, 412-19.

Iranian Health CoRps. Report (1969). Ministry of Health, Teheran, Iran.

LIDWELL, O. M. (1959). Apparatus for phage-typing of Staphylococcus aureus. Monthly Bulletin of the Ministry of Health and the Public Health Laboratory Service 18, 49-52.

Saragea, Alice \& Maximesco, P. (1966). Phage typing of Corynebacterium diphtheriae. Bulletin of the World Health Organization 35, 681-9.

Tahernia, A. C. \& Motamed, F. (1969). Diphtheria-still lethal. Clinical Pediatrics 8, 508-11.

ZAMIRI, I. (1970). Diphtheria today. Lancet i, 1222-3. 